\title{
Effect of osmotic stress and seed priming on wheat seed germination traits
}

\section{Navin Chander Gahtyari ${ }^{1 *}$, J. P. Jaiswal ${ }^{1}$, Mohammed Talha ${ }^{1}$, Rakesh Choudhary ${ }^{1}$, Meenakshi Uniyal ${ }^{1}$ and Naveen Kumar $^{2}$}

\author{
${ }^{1}$ Department of Genetics and Plant Breeding, G.B. Pant University of Agriculture and \\ Technology, Pantnagar, Uttarakhand-263145, India \\ ${ }^{2}$ Department of Molecular Biology and Genetic Engineering, G.B. Pant University of Agriculture \\ and Technology, Pantnagar, Uttarakhand - 263145, India
}

*Corresponding author

\begin{tabular}{|c|c|}
\hline & A B S T R A C T \\
\hline & $\begin{array}{l}\text { A study was undertaken to evaluate the effect of increasing osmotic stress on various } \\
\text { germination traits along with the capability of hydropriming and hormonal priming to }\end{array}$ \\
\hline Keywords & $\begin{array}{l}\text { counteract it. Seeds of five wheat genotypes were tested at four different stress levels } \\
\text { viz., } 0 \text { bar, }-3 \text { bar, }-9 \text { bar and }-15 \text { bar created using PEG- } 6000 \text {, either seed primed with }\end{array}$ \\
\hline $\begin{array}{l}\text { Bars, genotypes, } \\
\text { gibberellic acid, } \\
\text { hydropriming, } \\
\text { osmotic stress }\end{array}$ & $\begin{array}{l}\text { water (hydropriming) or with } \mathrm{GA}_{3} \text { (Gibberellic acid) @ } 50 \mathrm{ppm} \text { in a completely } \\
\text { randomized design with three replications. With increase in osmotic stress from } 0 \text { bar } \\
\text { to }-15 \mathrm{bar} \text {, germination traits like germination percentage }(81.5 \% \text { to } 15 \%) \text {, plumule } \\
\text { length }(7.8 \mathrm{~cm} \text { to } 0.4 \mathrm{~cm}) \text {, number of lateral roots }(5 \text { to } 1.9) \text {, fresh weight }(124 \mathrm{mg} \text { to }\end{array}$ \\
\hline Article Info & bar $\mathrm{GA}_{3}$ treatment was more effective for enhancing germination percentage $(11.5 \%$ \\
\hline $\begin{array}{l}\text { Accepted: } \\
\text { 08 March } 2017 \\
\text { Available Online: } \\
10 \text { April } 2017\end{array}$ & $\begin{array}{l}\text { and } 9.5 \% \text { increase over hydroprimed) whereas at higher stress levels i.e. }-9 \text { and }-15 \\
\text { bar hydropriming was found to be better than } \mathrm{GA}_{3} \text { treatment }(8 \% \text { and } 6 \% \text { increase } \\
\left.\text { over } \mathrm{GA}_{3}\right) \text {. Average germination percentage at all stress levels revealed PBW } 175 \\
(55.00 \%) \text {, C } 306(54.69 \%) \text { and MACS } 6272(53.13 \%) \text { as osmotic stress tolerant }\end{array}$ \\
\hline & $\begin{array}{l}\text { group and UP } 2828(50.63 \%) \text { and K } 1016(49.06 \%) \text { as osmotic stress susceptible } \\
\text { group. }\end{array}$ \\
\hline
\end{tabular}

\section{Introduction}

Wheat is an important world crop which is $1 \mathrm{st}$ in terms of area (221.6 million hectares) and $3^{\text {rd }}$ in terms of production (728.9 million tonnes) out of three major cereal crops i.e. maize, rice and wheat in the world (FAO, 2014). In India wheat is grown in 30.23 million hectare area with 93.50 million tonne production (Anon., 2016). Drought is one the major abiotic stresses which affects wheat production and reduces yield.
Agriculture drought can be defined as the limitation of moisture during various crop stages which adversely affects the average crop production. Seed germination is the first step in the life cycle of any crop plant and unavailability of moisture during germination severely affects the germination traits and yield (Ahmad et al., 2009). Osmotic stress in laboratory conditions can be created through the use of PEG-6000. PEG - 6000 is high molecular weight compounds, which 
decreases the water potential and thus makes it less available to germinating seed and it is preferable in use due to its non - toxic and non - permeable ability into the seeds (Gupta et al., 1993).

Seed priming is the application of natural or synthetic compounds to seed before germination. Seed priming increases the physiological and biochemical processes necessary for enhancing seed germination and associated traits. Seeds are hydrated to a point that germination processes start but radicle does not emerge out (Bradford, 1986). Hydropriming is the soaking of seed in sterilized distilled water at a specific temperature for a specific duration of time. It increases the biochemical and physiological processes in a seed, thus enhancing the germination capabilities in comparison to non-primed seeds. Hydropriming effects for germination trait enhancement has been reported by Rashid et al., 2004 in mungbean, Janmohammadi et al., 2008 in maize and Caseiro et al., 2004 in onion.

Phytohormones play an important role in the germinating seeds. The germinating seed has altered levels of abscisic acid and gibberellic acid $\left(\mathrm{GA}_{3}\right)$ (Seo et al., 2009) in comparison to non-germinating seeds. Osmotic stress decreases the cytokinin and $\mathrm{GA}_{3}$ levels in seeds whereas abscisic acid increases (Kaur et al., 1998). Hence, external use of $\mathrm{GA}_{3}$ and other phytohormones has been reported by many researchers with different optimum doses e.g. $50 \mathrm{ppm}$ for 24 hours in wheat (Abnavi and Ghobadi, 2012), $0.00001 \mathrm{mM}$ Salicylic acid in wheat (Movaghatian and Khorsandi, 2013), $6 \mu \mathrm{M}$ of $\mathrm{GA}_{3}$ and kinetin in chickpea (Kaur et al., 1998) etc. Thus, a study was undertaken to study the effect of increasing osmotic stress on seed germination traits and to check whether there exist any genotypic and treatment variations for different germination traits. These differences can be further utilized for screening purpose to have better tolerant genotypes for drought.

\section{Methods and Materials}

The experiment was conducted at the Grain quality \& Molecular marker laboratory of wheat; Department of Genetics and Plant Breeding, G. B. P. U. A \& T, Pantnagar. Five genotypes of wheat viz., C 306, PBW 175, MACS 6272, UP 2828 and K 1016 were tested for germination traits at four different osmotic stress levels. The five used genotypes were cultivated varieties. Before giving the treatment (phytohormonal or hydropriming), the wheat seed is disinfected with $0.1 \%$ solution of $\mathrm{HgCl}_{2}$ for 5 minutes. Thereafter, seeds were pre-soaked for a period of 24 hours at $25 \pm 1{ }^{\circ} \mathrm{C}$ either with hydropriming (pre-soaking with distilled water) or with phytohormonal treatment (pre-soaking with $\mathrm{GA}_{3}$ solution made of $50 \mathrm{ppm}$ ).

\section{Preparation of osmotic solution}

As per the equation by Michel and Kaufmann (1973) amount of PEG (in grams) to be dissolved in distilled water was calculated as per the following equation:

$\psi=-\left(1.18 \times 10^{-2}\right) C-\left(1.18 \times 10^{-4}\right) C^{2}+\left(2.67 \times 10^{-4}\right) C T+\left(8.39 \times 10^{-7}\right) C^{2} T$

Here, $\psi=$ osmotic potential in bars.

$\mathrm{C}=$ concentration of PEG-6000 in g/litre of $\mathrm{H}_{2} \mathrm{O}$.

$\mathrm{T}=$ Temperature in ${ }^{\circ} \mathrm{C}$.

The four osmotic stress levels used were 0 bar, -3 bar, -9 bar and -15 bar. The concentrations of PEG - 6000 were calculated for -3 bar, -9 bar and -15 bar osmotic solutions at temperature of $25^{\circ} \mathrm{C}$. For 0 bar, distilled water was used. $151.38 \mathrm{~g}, 279.27 \mathrm{~g}$ and $367.64 \mathrm{~g}$ of PEG was dissolved in 1 litre of distilled water for preparing osmotic solution of -3 bar, -9 bar and -15 bar, respectively. 
Germination traits and recording of observations

The 24 hour pre-soaked seeds were then washed with distilled water and dried up using blotting paper at room temperature. 50 seeds of a particular genotype were spread on a filter paper (Whatman \# 41) in a petriplates and moistened with $10 \mathrm{ml}$ of either $0,-3,-9$ or -15 bar osmotic solution (Abnavi and Ghobadi, 2012). The experiment was conducted in a completely randomized design with three replications. Hence a total of 5 genotypes x 2 treatment $\times 4$ stress level $\times 3$ replication i.e. 120 petriplates was used for the experimentation. Data for seven germination traits viz. Germination percentage $(\%)$, radicle length $(\mathrm{cm})$, plumule length $(\mathrm{cm})$, no. of lateral roots, fresh weight of seedling (mg), dry weight of seedling (mg) and radicle/plumule ratio were taken from random 5 seedlings in each replication. A seed having $1 \mathrm{~mm}$ radicle was considered germinated and all seven traits were measured after 7 days. Germination percentage was calculated as no. of seed germinated on 7th Day/Total no. of seeds (Ashraf et al., 1978). Radicle length, plumule length was measured in centimetres $(\mathrm{cm})$. Fresh weight and dry weight was measured in milligrams $(\mathrm{mg})$ and whole seedling fresh and dry weight is considered. The experimental results were analysed using OPSTAT software (Sheoran et al., 1998) and the treatment means were compared using least significant difference (CD value) of OPSTAT (Sheoran et al., 1998). The Karl pearson's simple correlation coefficient was also computed between different germination traits using OPSTAT (Sheoran et al., 1998).

\section{Results and Discussion}

\section{Analysis of variance}

Most of the single factors (treatment, osmotic stress and genotypes) and interactions were found significant in ANOVA analysis revealing that there is considerable variation between genotypes, stress levels and treatments for seed germination traits. However there were insignificant results like insignificant treatments for no. of lateral roots, insignificant interaction of treatments $x$ osmotic stress levels for dry weight, and insignificant interaction of treatments $x$ genotypes and treatment $\mathrm{x}$ stress levels $\mathrm{x}$ genotypes for germination percentage and radicle/plumule ratio (Table 1).

\section{Germination percentage (\%)}

The germination percentage decreased with increasing stress level (Fig. 1). Highest average germination of $81.5 \%$ was recorded with $\mathrm{GA}_{3}$ treatment at 0 bar stress which lowered to average germination percentage of $15 \%$ at -15 bars. At 0 and -3 bar $\mathrm{GA}_{3}$ treatment was more effective for enhancing germination percentage with an increase of $11.5 \%$ and $9.5 \%$ respectively, as compared to hydroprimed seeds. However, at high stress levels i.e. -9 and -15 bar hydropriming was found to be better than $\mathrm{GA}_{3}$ treatment for enhancing seed germination. Hydroprimed seed showed $8 \%$ and 6\% increase in germination percentage as compared to $\mathrm{GA}_{3}$ seeds at -9 and -15 bar stress level respectively. The four stress levels differed significantly from each other with highest average germination percentage at 0 bar $(75.8$ $\%)$ with a gradual decrease in germination at 3 bar $(67.3 \%),-9(49.0 \%)$ and $-15 \operatorname{bar}(18.0$ $\%$ ) (Table 2).

There is no significant difference observed for hydroprimed and $\mathrm{GA}_{3}$ treated seeds for germination percentage. The highest germination percentage was observed for PBW $175(55.00 \%)$ which does not varied significantly from C $306(54.69 \%)$ and MACS $6272 \quad(53.13 \%)$ but differed significantly from other two genotypes viz., UP $2828(50.63 \%)$ and K $1016(49.06 \%)$ (Table 3). 
Radicle length, plumule length and radicle/plumle length ratio

For both radicle and plumule length, all single factors (treatments, stress levels and genotypes) and interactions (treatment $\mathrm{x}$ stress levels and treatment $x$ genotypes) were found to be significant. However, the stress level $x$ genotype interaction was significant for radicle length but insignificant for plumule length (Table 1).

Radicle length varied from $9.5 \mathrm{~cm}$ (K 1016 , GA3, -3 bar) to $1.0 \mathrm{~cm}$ (PBW 175, hydroprimed, -15 bar) with average radicle length varying from $7.8 \mathrm{~cm}\left(\mathrm{GA}_{3},-3 \mathrm{bar}\right)$ to $1.9 \mathrm{~cm}$ (hydroprimed, -15 bar). Average plumule length varied from $9.1 \mathrm{~cm}$ (hydroprimed, 0 bar) to $0.3 \mathrm{~cm}$ (hydroprimed, -15 bar). The four stress levels also differed significantly from each other with highest average radicle length at -3 bar $(5.9 \mathrm{~cm})$ followed by -9 bar $(4.9 \mathrm{~cm}), 0$ bar $(4.4 \mathrm{~cm})$ and -15 bar $(2.0 \mathrm{~cm})($ Table 2$)$. Plumule length decreased with increasing stress level for both hydroprimed and $\mathrm{GA}_{3}$ primed seed (Fig. 1, Table 2).Average plumule length showed gradual decrease from $7.8 \mathrm{~cm}(0 \mathrm{bar}), 6.1 \mathrm{~cm}$ (-3 bar), $0.7 \mathrm{~cm}$ ( -9 bar) to $0.4 \mathrm{~cm}$ (-15 bar).

Radicle length and plumule length showed an opposite effects for hormonal treatment. Whereas, average radicle length was always greater for $\mathrm{GA}_{3}$ treated seeds as compared to hydroprimed seeds at all stress levels, average plumule length showed the opposite trend and it was greater for hydroprimed seed as compared to $\mathrm{GA}_{3}$ treated seeds at all stress levels except at -15 bar where $\mathrm{GA}_{3}$ treated seeds showed mean plumule length of $0.4 \mathrm{~cm}$ against mean plumule length of $0.3 \mathrm{~cm}$ of hydroprimed seeds (Fig. 1, Table 2).

Radicle/plumule ratio varied from 36.4 (C $306, \mathrm{GA}_{3},-9$ bar $)$ to $0.2 \quad(\mathrm{~K} 1016$, Hydroprimed, 0 bar). The average radicle/plumule ratio varied from $21.3\left(\mathrm{GA}_{3}\right.$, 9 bar) to 0.4 (hydroprimed, 0 bar). The average radicle/plumule ratio was always greater for $\mathrm{GA}_{3}$ treated seed as compared to hydroprimed seeds at all four stress levels. The highest radicle/plumule ratio was observed at high stress levels i.e. -9 bar (15.7) and -15 bar (11.8) which decreased significantly at lower stress levels. At -3 bar it was 1.1 which further lowered to 0.6 at 0 bar (Table 2).

For radicle length, all five genotypes showed the similar pattern of $\mathrm{GA}_{3}$ treated seeds having larger radicle length as compared to hydroprimed seeds (Table 3). Hydroprimed and $\mathrm{GA}_{3}$ treated groups differed significantly from each other with $3.30 \mathrm{~cm}$ and $5.28 \mathrm{~cm}$ radicle length, respectively. The highest radicle length was observed for MACS 6272 $(4.56 \mathrm{~cm})$ which doesn't varied significantly from other three genotypes i.e. (C 306, PBW $175 \&$ K 1016) and the lowest is for UP 2828 $(3.61 \mathrm{~cm})$ which had significant lower radicle length from the other four genotypes. Hydroprimed and $\mathrm{GA}_{3}$ treated groups differed significantly from each other with $4.24 \mathrm{~cm}$ and $3.19 \mathrm{~cm}$ plumule length, respectively. The highest plumule length was observed for UP $2828(4.27 \mathrm{~cm})$ which was at par with C 306 $(3.75 \mathrm{~cm})$ but varied significantly from PBW $175(3.66 \mathrm{~cm})$, MACS $6272(3.55 \mathrm{~cm})$ and $\mathrm{K}$ $1016(3.33 \mathrm{~cm})$ (Table 3).

\section{Number of lateral roots}

ANOVA for no. of lateral roots revealed stress levels, genotypes and interactions, treatments $\mathrm{x}$ genotypes and stress levels $\mathrm{x}$ genotypes to have significant differences (Table 1).

No. of lateral roots varied from 6.7 (C 306, hydroprimed, 0 bar) to 1 (MACS 6272, $\mathrm{GA}_{3}$, 15 bar). No. of lateral roots decreased with increasing stress level (Fig 2). They were 
highest at 0 bar (5.0) lowered to 4.7 ( -3 bar), 3.2 ( -9 bar) and 1.9 at -15 bar with a gradual decrease.

There was no significant difference observed for hydroprimed and $\mathrm{GA}_{3}$ treated seeds for no. of lateral roots. The highest no. of lateral roots was observed for C 306 (3.95) which was at par with UP $2828(3.88 \mathrm{~cm})$ and $\mathrm{K}$ $1016(3.73 \mathrm{~cm})$ but varied significantly from MACS 6272 (3.48) and PBW 175 (3.33) (Table 3).

\section{Fresh weight and dry weight}

For both fresh weight and dry weight all single factors (treatment, stress levels and genotypes) and interactions (stress levels $\mathrm{x}$ genotypes and treatment $\mathrm{x}$ genotypes) were found to be significant. However, treatment $\mathrm{x}$ stress levels was significant for fresh weight but insignificant in case of dry weight (Table $1)$.

Fresh weight varied from $171.2 \mathrm{mg}$ (MACS 6272, $\mathrm{GA}_{3}, 0$ bar) to $52.7 \mathrm{mg}$ (UP 2828, hydroprimed, -15 bar) and dry weight varied from $18.0 \mathrm{mg}$ (C 306, $\mathrm{GA}_{3}, 0$ bar) to $56.8 \mathrm{mg}$ (MACS 6272, $\mathrm{GA}_{3},-15$ bar). The average fresh weight was always greater for $\mathrm{GA}_{3}$ treated seed as compared to hydroprimed seeds at all four stress levels.

Fresh weight showed a decreasing trend with highest average fresh weight at 0 bar (124 mg) which decreased significantly with increasing stress levels. At -3 bar it went down to $109.4 \mathrm{mg}$ followed by $88.9 \mathrm{mg}$ at -9 bar and $77.4 \mathrm{mg}$ at -15 bar (Table 2). The average dry weight showed a opposite trend w.r.t fresh weight and it increased with increasing stress level for both hydroprimed and $\mathrm{GA}_{3}$ primed seed. The highest dry weight was observed at -15 bar $(40.5 \mathrm{mg})$ which decreased significantly to $34.4 \mathrm{mg}$ (-9 bar), $28.2 \mathrm{mg}$ (-3 bar) and $24.7 \mathrm{mg}$ (Obar) (Fig. 1, Table 2).
Whereas hydroprimed and $\mathrm{GA}_{3}$ treated groups differed significantly from each other for fresh weigh $(83.77 \mathrm{mg}$ and $116.07 \mathrm{mg}$ respectively), for dry weight the difference was insignificant $(31.45 \mathrm{mg} \& 32.45 \mathrm{mg}$ respectively). The highest fresh weight was observed for MACS 6272 (106.05 mg) which was at par with K 1016 (103.30 mg) and UP $2828(100.02 \mathrm{mg})$ but varied significantly from PBW 175 (95.68 mg) and C 306 (94.55 $\mathrm{mg}$ ). For dry weight, the lowest weight was observed for UP 2828 (28.92 mg) which was at par with C $306(29.48 \mathrm{mg})$ but varied significantly from PBW 175 (32.78 mg), K 1016 (33.94 mg) and MACS 6272 (34.64 mg) (Table 3).

\section{Correlation between the germination traits}

At no stress i.e. 0 bar germination percentage was having significant correlation with radicle length $\left(0.83^{* *}\right)$, plumule length $\left(0.65^{*}\right)$, fresh weight $\left(0.86^{* *}\right)$ and radicle/plumule length ratio $\left(0.83^{* *}\right)$. But with an increase in stress level this significant positive correlation faded away to non-significant correlation with minor exceptions at -3 bar for radicle length $(0.69 *)$ and radicle to plumule length ratio $(0.73 *)$ (Table 4$)$.

The consistency of significant positive correlation at three consecutive stress levels was found for only two correlation coefficients. Firstly, the correlation coefficient between fresh weight and radicle length was found significant at 0 bar $(0.77 * *)$, -3 bar $\left(0.70^{*}\right)$ and -9 bar $\left(0.85^{* *}\right)$ and secondly, correlation coefficient between radicle length and radicle/plumule length ratio was found significant at 0 bar $\left(0.96^{* *}\right),-3$ bar $(0.79 * *)$ and -9 bar $(0.65 *)$ (Table 4$)$.

The correlation of plumule length with radicle to plumule length ratio was also positive and high (>0.60) at 0 bar, -3 bar and -9 bar. 
Figure 1: Graphical representation of seven germination traits on five genotypes of wheat with hydropriming and $\mathrm{GA}_{3}$ treatment. C ( 0 bar): seed hydroprimed at 0 bar stress level; $\mathrm{GA}\left(0\right.$ bar): $\mathrm{GA}_{3}$ priming at 0 bar stress level; C ( -3 bar): seed hydroprimed at -3 bar stress level; GA ( -3 bar): $\mathrm{GA}_{3}$ priming at -3 bar stress level; $\mathrm{C}$ ( -9 bar): seed hydroprimed at -9 bar stress level; GA (-9 bar): $\mathrm{GA}_{3}$ priming at -9 bar stress level; $\mathrm{C}$ ( -15 bar): seed hydroprimed at -15 bar stress level; GA (-15 bar): $\mathrm{GA}_{3}$ priming at -15 bar stress level.
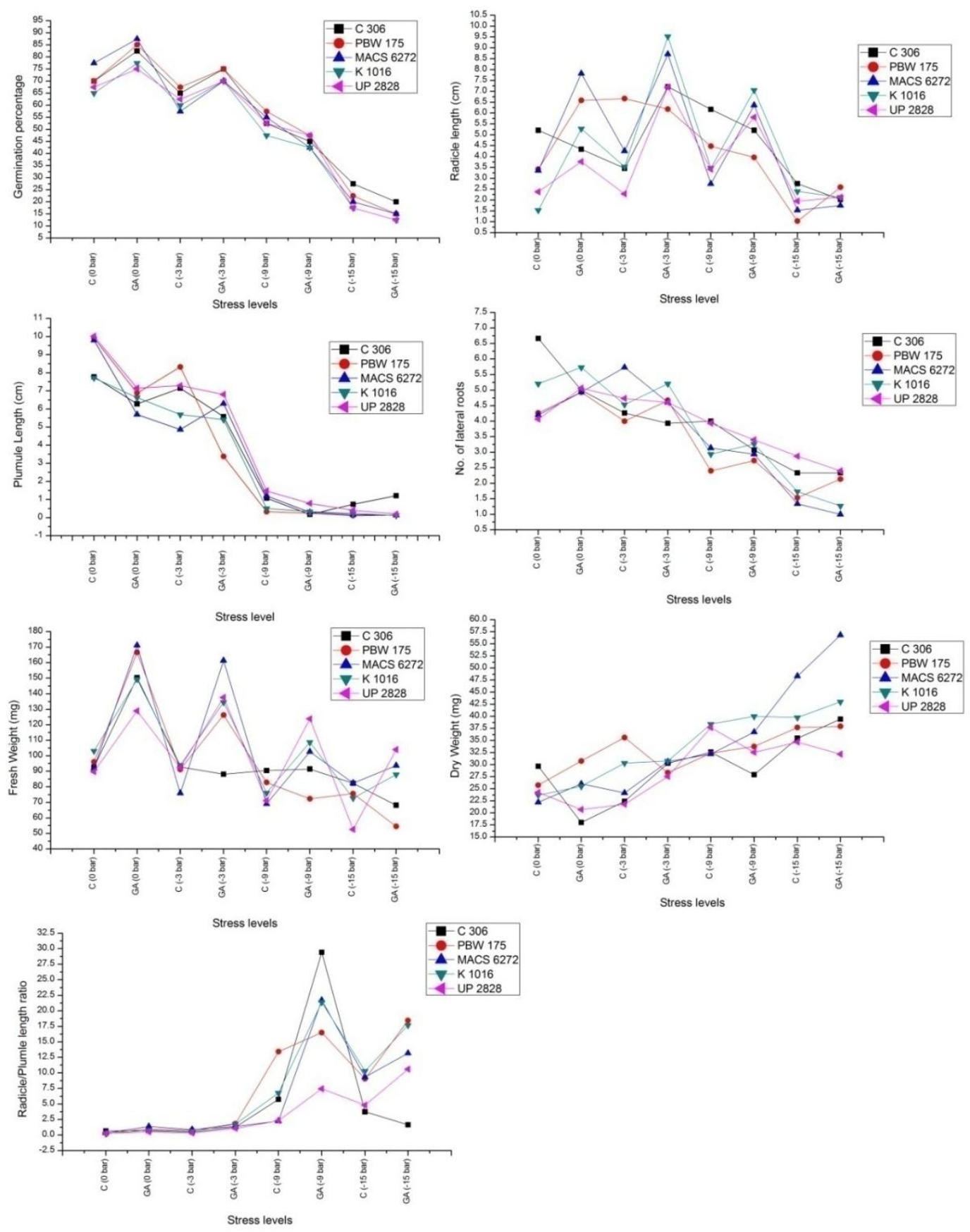
Table.1 Analysis of variance (mean sum of square) for seven germination traits in wheat

\begin{tabular}{|c|c|c|c|c|c|c|c|c|}
\hline Source of Variation & $\mathrm{DF}$ & Germination percentage & Radicle length & Plumule length & No. of lateral roots & Fresh weight & Dry weight & Radicle/Plumule ratio \\
\hline $\begin{array}{c}\text { Factor A } \\
\text { (hydroprimed/GA })\end{array}$ & 1 & $91.88^{*}$ & $117.67 * * *(0.33)$ & $33.28 * * *(0.33)$ & 0.03 & $31318.18 * * *(4.91)$ & $29.84 *$ & $510.59 * *(2.90)$ \\
\hline Factor B (stress levels) & 3 & $19606.25 * * *(3.39)$ & $80.50 * * *(0.46)$ & $429.38 * * *(0.47)$ & $60.98 * * *(0.29)$ & $12993.83 * * *(6.95)$ & $1471.11 * * *(3.32)$ & $1741.82 * * *(4.11)$ \\
\hline Interaction A X B & 3 & $775.63 * * *(4.81)$ & $16.39 * * *(0.65)$ & $8.99 * * *(0.66)$ & $0.30^{*}$ & $3564.63 * * *(9.83)$ & 28.45 & $184.23 * *(5.81)$ \\
\hline Factor C (Genotypes) & 4 & $160.55^{* *}(3.80)$ & $3.66 * * *(0.51)$ & $2.88 * *(0.52)$ & $1.66^{* * * *}(0.33)$ & $574.68 * *(7.77)$ & $162.88 * *(3.71)$ & $143.36^{* *}$ \\
\hline Interaction A X C & 4 & 3.98 & $10.65 * * *(0.73)$ & $2.07 * *(0.74)$ & $1.47 * *(0.46)$ & $1971.38 * * *(10.99)$ & $52.79 *$ & 18.37 \\
\hline Interaction B X C & 12 & $52.73 *$ & $3.35 * * *(1.03)$ & $1.28 *$ & $1.63 * * *(0.65)$ & $482.65 * *(15.54)$ & $101.14 * *(7.42)$ & $139.88 * *(9.18)$ \\
\hline Interaction A X B X C & 12 & 11.17 & $4.68 * * *(1.45)$ & $2.97 * * *(1.48)$ & $0.64 * *(0.92)$ & $739.59 * * *(21.98)$ & $42.80^{*}$ & 33.09 \\
\hline Error & 80 & 43.75 & 0.8 & 0.83 & 0.32 & 182.85 & 41.67 & 63.82 \\
\hline Total & 119 & & & & & & & \\
\hline
\end{tabular}

“*”, “**”, “***” Significant at 5\%, $1 \%$ and $0.01 \%$ respectively. The value in parentheses ( ) represent C.D. (least significant difference) value at $1 \%$ level of significance

Table. 2 Mean comparison of 5 wheat genotypes at 4 different osmotic stress level with hydropriming and $\mathrm{GA}_{3}$ treatment on different germination traits.

\begin{tabular}{|c|c|c|c|c|c|c|c|c|c|c|c|c|c|c|c|c|c|c|c|c|c|c|c|c|c|c|c|c|c|}
\hline \multirow{2}{*}{ Genotypes } & \multirow{2}{*}{ Treatment } & \multicolumn{7}{|c|}{0 bar } & \multicolumn{7}{|c|}{$-3 \mathrm{bar}$} & \multicolumn{7}{|c|}{-9 bar } & \multicolumn{7}{|c|}{-15 bar } \\
\hline & & $\overline{G P}$ & RL & PL & LR & FW & DW & $\mathrm{L} / \mathrm{PL}$ & $\overline{G P}$ & $\overline{R L}$ & PL & LR & FW & DW & RL/PL & $\mathrm{GP}$ & RL & PL & LR & FW & $\overline{\mathrm{DW}}$ & RL/PL & GP & RL & PL & LR & FW & $\overline{D W}$ & $\mathrm{RL} / \mathrm{PL}$ \\
\hline \multirow{3}{*}{ C 306} & Hydroprimed & 70.0 & 5.2 & 7.8 & 6.7 & 92.8 & 29.7 & 0.7 & 65.0 & 3.5 & 7.2 & 4.3 & 92.8 & 22.4 & 0.6 & 52.5 & 6.2 & 1.1 & 4.0 & 90.5 & 32.6 & 24.5 & 27.5 & 2.8 & 0.7 & 2.3 & 82.2 & 35.5 & 11.8 \\
\hline & & 82.5 & 4.3 & 6.3 & 5.0 & 150.3 & 18.0 & 0.7 & 5.0 & 7.2 & 5.6 & 3.9 & 88.1 & 30.3 & 1.3 & 45.0 & 5.2 & 0.2 & 3.1 & 91.5 & 27.9 & 36.4 & 20.0 & 2.0 & 1.2 & 2.3 & 68.1 & 39.5 & 6.6 \\
\hline & Aver & 76.3 & 4.8 & 7.1 & 5.9 & 121.6 & 23.9 & 0.7 & 70.0 & 5.4 & 6.4 & 4.1 & 90.5 & 6.4 & 1.0 & 48.8 & 5.7 & 0.7 & 3.6 & 91.0 & 30.3 & 30.5 & 23.8 & 2.4 & 1.0 & 2.3 & 75.2 & 37.5 & 9.2 \\
\hline \multirow{3}{*}{ PBW 175} & Hydroprime & 70.0 & 3.4 & 9.9 & 4.3 & 96.1 & 25.8 & 0.3 & 67.5 & 6.7 & 8.3 & 4.0 & 91.2 & 35.6 & 0.8 & 57.5 & 4.5 & 0.3 & 2.4 & 82.8 & 32 & 13.7 & 22.5 & 1.0 & 0.1 & .5 & 75.6 & 37.7 & 9.2 \\
\hline & $\mathrm{GA}_{3}$ & 85.0 & 6.6 & 6.9 & 4.9 & 166.6 & 30.8 & 1.0 & 75.0 & 6.2 & 3.4 & 4.7 & 126.3 & 28.3 & 1.9 & 47.5 & 4.0 & 0.2 & 2.7 & 72.2 & 33.8 & 17.3 & 15.0 & 2.6 & 0.1 & 2.1 & 54.5 & 37.9 & 19.1 \\
\hline & Aver & 77.5 & 5.0 & 8.4 & 4.6 & 131.4 & 28.3 & 0.7 & 71.3 & 6.5 & 5.9 & 4.4 & 108.8 & 32.0 & 1.4 & 52.5 & 4.3 & 0.3 & 2.6 & 77.5 & 33.1 & 15.5 & 18.8 & 1.8 & 0.1 & 1.8 & 65.1 & 37.8 & 14.2 \\
\hline MACS & Hydrop & 77.5 & 3.4 & 9.8 & 4.2 & 920 & 22.2 & 03 & 7.5 & 4.3 & 4.9 & 5.7 & 76.0 & 24.1 & 0.9 & 55.0 & 2.7 & 1.2 & 3.1 & 69.0 & 32.2 & 3.1 & 20.0 & 1.5 & 0.2 & 1. & 82.5 & 48.3 & 12.4 \\
\hline \multirow[t]{2}{*}{6272} & & 87.5 & 7.8 & 5.7 & 4.9 & 171.2 & 26.0 & 1.5 & 70.0 & 8.7 & 6.3 & 4.6 & 161.4 & 30.6 & 1. & 42.5 & 6.4 & 0.3 & 2.9 & 102.6 & 36.7 & 23.1 & 15.0 & 1.8 & 0.1 & 1.0 & 93.7 & 56.8 & 14.2 \\
\hline & Average & 82.5 & 5.6 & 7.8 & 4.6 & 131.6 & 24.1 & 0.9 & 63.8 & 6.5 & 5.6 & 5.2 & 118.7 & 27.4 & 1.2 & 48.8 & 4.6 & 0.8 & 3.0 & 85.8 & 34.5 & 13.1 & 17.5 & 1.7 & 0.2 & 1.2 & 88.1 & 52.6 & 13.3 \\
\hline \multirow{3}{*}{ K 1016} & Hydropr & 65.0 & 1.5 & 7.7 & 5.2 & 103.1 & 23.7 & & 60.0 & 3.5 & 5.7 & 4.5 & & 202 & & 47.5 & 3.5 & 0.5 & 2.9 & 76.1 & 38.4 & 60 & 17.5 & 2.4 & 0.2 & 1.7 & 72.8 & 39.8 & 11.2 \\
\hline & & 7.5 & 5.3 & 6.6 & 5.7 & 149.3 & 25 & 0 & 70.0 & 9.5 & 5.4 & 5.2 & 134.5 & $J$ & 1. & 42.5 & 7.1 & 0.3 & 3.3 & 108.7 & 4( & 21.3 & 12.5 & 2.1 & 0.1 & 1.3 & 88.0 & 43.0 & 18.5 \\
\hline & Aver: & 71.3 & 3.4 & 7.2 & 5.5 & 126.2 & 24.6 & 0.5 & 65.0 & 6.5 & 5.6 & 4.9 & 114.3 & 30.6 & 1.3 & 45.0 & 5.3 & 0.4 & 3.1 & 92.4 & 39.2 & 14.1 & 15.0 & 2.3 & 0.2 & 1.5 & 80.4 & 41.4 & 14.9 \\
\hline \multirow{3}{*}{ UP 2828} & Hydroprimed & 67.5 & 2.4 & 10.0 & 4.1 & 89.8 & 24.2 & 0.2 & 62.5 & 2.3 & 7.3 & 4.7 & 92.3 & 21.8 & 0. & 52.5 & 3.4 & 1.5 & 3.9 & 71.1 & 37.7 & 2.3 & 17.5 & 2.0 & 0.4 & 2.9 & 52.7 & 34.7 & 4.8 \\
\hline & & 750 & 3.8 & 7.2 & 5.1 & 128.9 & 20. & 0 . & 70.0 & 7.2 & 6.8 & 4.6 & 13 & 27 & 1 & 47.5 & 5.8 & 0.8 & 3.4 & 123.9 & 32 & 8. & 12.5 & 2.1 & 0.2 & 2.4 & 104.0 & 32.2 & 10.7 \\
\hline & $\Delta$ & 71.3 & 3.1 & 8.6 & 4.6 & 109.4 & 22.5 & 0 & 66.3 & 4.8 & 7.1 & 4.7 & 115.0 & 24.7 & 0.7 & 50.0 & 4.6 & 1.2 & 3.7 & 97.5 & 35.1 & 5.3 & 15.0 & 2.1 & 0.3 & 2.7 & 78.4 & 33.5 & 7.8 \\
\hline \multirow{3}{*}{ Average } & Hydroprimed & 70.0 & 3.2 & 9.1 & 4.9 & 94.7 & 25.1 & 0.4 & 62.5 & 4.0 & 6.7 & 4.7 & 89.3 & 26.9 & 0.7 & 53.0 & 4.1 & 0.9 & 3.3 & 77.9 & 34.7 & 10.1 & 21.0 & 1.9 & 0.3 & 2.0 & 73.2 & 39.2 & 9.9 \\
\hline & & 81.5 & 5.6 & 6.5 & 5.1 & 153.3 & 24.2 & 0.9 & 72.0 & 7.8 & 5.5 & 4.6 & 129.6 & 29.5 & 1.5 & 45.0 & 5.7 & 0.4 & 3.1 & 99.8 & 34.2 & 21.3 & 15.0 & 2.1 & 0.4 & 1.8 & 81.7 & 41.9 & 13.8 \\
\hline & Average & 75.8 & 4.4 & 7.8 & 5.0 & 124.0 & 24.7 & 0.7 & 67.3 & 5.9 & 6.1 & 4.7 & 109.5 & 28.2 & 1.1 & 49.0 & 4.9 & 0.7 & 3.2 & 88.9 & 34.5 & 15.7 & 18.0 & 2.0 & 0.4 & 1.9 & 77.5 & 40.6 & 11.9 \\
\hline
\end{tabular}

GP - Germination percentage (\%), RL - Radicle length (cm), PL - Plumule length (cm), LR - No. of lateral roots, FW - Fresh Weight, DW - Dry Weight, RL/PL - Radicle length/Plumule length ratio. 
Table.3 Comparison of hydropriming vs $\mathrm{GA}_{3}$ treatment on 5 genotypes of wheat with overall average at 4 stress level on different germination traits

\begin{tabular}{|c|c|c|c|c|c|c|c|c|}
\hline Genotypes & Treatment & GP & RL & PL & $\mathbf{L R}$ & FW & DW & RL/PL \\
\hline \multirow{3}{*}{ C 306} & Hydroprimed & 53.75 & 4.43 & 4.20 & 4.33 & 89.58 & 30.05 & 9.40 \\
\hline & $\mathrm{GA}_{3}$ & 55.63 & 4.68 & 3.33 & 3.58 & 99.50 & 28.93 & 11.25 \\
\hline & Average & 54.69 & 4.55 & 3.76 & 3.95 & 94.54 & 29.49 & 10.33 \\
\hline \multirow{3}{*}{ PBW 175} & Hydroprimed & 54.38 & 3.90 & 4.65 & 3.05 & 86.43 & 32.88 & 6.00 \\
\hline & $\mathrm{GA}_{3}$ & 55.63 & 4.85 & 2.65 & 3.60 & 104.90 & 32.70 & 9.83 \\
\hline & Average & 55.00 & 4.38 & 3.65 & 3.33 & 95.66 & 32.79 & 7.91 \\
\hline \multirow{3}{*}{ MACS 6272} & Hydroprimed & 52.50 & 2.98 & 4.03 & 3.58 & 79.88 & 31.70 & 4.18 \\
\hline & $\mathrm{GA}_{3}$ & 53.75 & 6.18 & 3.10 & 3.35 & 132.23 & 37.53 & 10.05 \\
\hline & Average & 53.13 & 4.58 & 3.56 & 3.46 & 106.05 & 34.61 & 7.11 \\
\hline \multirow{3}{*}{ K 1016} & Hydroprimed & 47.50 & 2.73 & 3.53 & 3.58 & 86.50 & 33.05 & 4.73 \\
\hline & $\mathrm{GA}_{3}$ & 50.63 & 6.00 & 3.10 & 3.88 & 120.13 & 34.83 & 10.63 \\
\hline & Average & 49.06 & 4.36 & 3.31 & 3.73 & 103.31 & 33.94 & 7.68 \\
\hline \multirow{3}{*}{ UP 2828} & Hydroprimed & 50.00 & 2.53 & 4.80 & 3.90 & 76.48 & 29.60 & 1.90 \\
\hline & $\mathrm{GA}_{3}$ & 51.25 & 4.73 & 3.75 & 3.88 & 123.60 & 28.25 & 5.15 \\
\hline & Average & 50.63 & 3.63 & 4.28 & 3.89 & 100.04 & 28.93 & 3.53 \\
\hline \multirow{3}{*}{ Average } & Hydroprimed & 51.63 & 3.30 & 4.25 & 3.73 & 83.78 & 31.48 & 5.28 \\
\hline & $\mathbf{G A}_{3}$ & 53.38 & 5.30 & 3.20 & 3.65 & 116.10 & 32.45 & 9.38 \\
\hline & Average & 52.50 & 4.30 & 3.73 & 3.69 & 99.94 & 31.96 & 7.33 \\
\hline
\end{tabular}

GP - Germination percentage (\%), RL - Radicle length (cm), PL - Plumule length (cm), LR - No. of lateral roots, FW - Fresh Weight, DW - Dry Weight, RL/PL - Radicle length/Plumule length ratio

Table.4 Correlation coefficient (r) between germination traits at four different stress levels with pooled data for five wheat genotypes

\begin{tabular}{|c|c|c|c|c|c|c|c|c|}
\hline & & GP & RL & PL & LR & FW & DW & RL/PL \\
\hline \multirow{4}{*}{ GP } & $0 \mathrm{bar}$ & \multirow{4}{*}{1} & & & & & & \\
\hline & -3 bar & & & & & & & \\
\hline & -9 bar & & & & & & & \\
\hline & $-15 \mathrm{bar}$ & & & & & & & \\
\hline \multirow{4}{*}{ RL } & 0 bar & $0.83 * *$ & \multirow{4}{*}{1} & & & & & \\
\hline & -3 bar & $0.69 *$ & & & & & & \\
\hline & -9 bar & $0.59^{\mathrm{NS}}$ & & & & & & \\
\hline & $-15 \mathrm{bar}$ & $0.03^{\mathrm{NS}}$ & & & & & & \\
\hline \multirow{4}{*}{ PL } & 0 bar & $0.65^{*}$ & $0.67 *$ & \multirow{4}{*}{1} & & & & \\
\hline & -3 bar & $0.23^{\mathrm{NS}}$ & $0.15^{\mathrm{NS}}$ & & & & & \\
\hline & -9 bar & $0.55^{\mathrm{NS}}$ & $0.40^{\mathrm{NS}}$ & & & & & \\
\hline & -15 bar & $0.48^{\mathrm{NS}}$ & $0.29^{\mathrm{NS}}$ & & & & & \\
\hline \multirow{4}{*}{ LR } & $0 \mathrm{bar}$ & $0.03^{\mathrm{NS}}$ & $0.33^{\mathrm{NS}}$ & $0.53^{\mathrm{NS}}$ & \multirow{4}{*}{1} & & & \\
\hline & -3 bar & $0.47^{\mathrm{NS}}$ & $0.04^{\mathrm{NS}}$ & $0.46^{\mathrm{NS}}$ & & & & \\
\hline & -9 bar & $0.03^{\mathrm{NS}}$ & $0.17^{\mathrm{NS}}$ & $0.75^{*}$ & & & & \\
\hline & -15 bar & $0.18^{\mathrm{NS}}$ & $0.43^{\mathrm{NS}}$ & $0.52^{\mathrm{NS}}$ & & & & \\
\hline \multirow{4}{*}{ FW } & 0 bar & $0.86 * *$ & $0.77 * *$ & $0.87 * *$ & $0.15^{\mathrm{NS}}$ & \multirow{4}{*}{1} & & \\
\hline & -3 bar & $0.54^{\mathrm{NS}}$ & $0.70^{*}$ & $0.15^{\mathrm{NS}}$ & $0.06^{\mathrm{NS}}$ & & & \\
\hline & -9 bar & $0.55^{\mathrm{NS}}$ & $0.85^{* *}$ & $0.29^{\mathrm{NS}}$ & $0.12^{\mathrm{NS}}$ & & & \\
\hline & -15 bar & $0.21^{\mathrm{NS}}$ & $0.14^{\mathrm{NS}}$ & $0.24^{\mathrm{NS}}$ & $0.46^{\mathrm{NS}}$ & & & \\
\hline \multirow{4}{*}{ DW } & 0 bar & $0.03^{\mathrm{NS}}$ & $0.47^{\mathrm{NS}}$ & $0.01^{\mathrm{NS}}$ & $0.36^{\mathrm{NS}}$ & $0.07^{\mathrm{NS}}$ & \multirow{4}{*}{1} & \\
\hline & -3 bar & $0.43^{\mathrm{NS}}$ & $0.67 *$ & $0.07^{\mathrm{NS}}$ & $0.38^{\mathrm{NS}}$ & $0.27^{\mathrm{NS}}$ & & \\
\hline & -9 bar & $0.31^{\mathrm{NS}}$ & $0.10^{\mathrm{NS}}$ & $0.08^{\mathrm{NS}}$ & $0.13^{\mathrm{NS}}$ & $0.02^{\mathrm{NS}}$ & & \\
\hline & $-15 \mathrm{bar}$ & $0.17^{\mathrm{NS}}$ & $0.33^{\mathrm{NS}}$ & $0.27^{\mathrm{NS}}$ & $0.82 * *$ & $0.29^{\mathrm{NS}}$ & & \\
\hline \multirow{4}{*}{ RL/PL } & 0 bar & $0.83 * *$ & $0.96^{* *}$ & $0.78^{* *}$ & $0.31^{\mathrm{NS}}$ & $0.83^{* *}$ & $0.34^{\mathrm{NS}}$ & \multirow{4}{*}{1} \\
\hline & -3 bar & $0.73^{*}$ & $0.79 * *$ & $0.67 *$ & $0.16^{\mathrm{NS}}$ & $0.61^{\mathrm{NS}}$ & $0.42^{\mathrm{NS}}$ & \\
\hline & -9 bar & $0.50^{\mathrm{NS}}$ & $0.65^{*}$ & $0.62^{\mathrm{NS}}$ & $0.09^{\mathrm{NS}}$ & $0.34^{\mathrm{NS}}$ & $0.38^{\mathrm{NS}}$ & \\
\hline & -15 bar & $0.40^{\mathrm{NS}}$ & $0.31^{\mathrm{NS}}$ & $0.52^{\mathrm{NS}}$ & $0.54^{\mathrm{NS}}$ & $0.22^{\mathrm{NS}}$ & $0.35^{\mathrm{NS}}$ & \\
\hline
\end{tabular}

GP - Germination percentage (\%), RL - Radicle length (cm), PL - Plumule length (cm), LR - No. of lateral roots, FW - Fresh Weight, DW -

Dry Weight, RL/PL - Radicle length/Plumule length ratio. “**”, “*”, Significant at 1\% and 5\% level of probability, respectively. 
Osmotic stress during the seed germination is said to have detrimental effects on various seed germination traits (Heikal et al., 1982). Low concentration of hormonal treatment viz., Salicylic acid (Movaghatian and Khorsandi, 2013) and $\mathrm{GA}_{3}$ (Abnavi and Ghobadi, 2012; Heikal et al., 1982) are better suited to counterattack the ill effects of osmotic stress rather than medium or high doses of these hormones. Also, low concentration of salicylic acid $(0.00001 \mathrm{mM})$ is effective for increasing germination percentage at high stress level i.e. at -6 and -9 bars (Movaghatian and Khorsandi, 2013) and hence $\mathrm{GA}_{3}$ can also be used as done in this study with its most optimum dose reported i.e. $50 \mathrm{ppm}$ for 24 hours pre-soaking (Abnavi and Ghobadi, 2012).

With increase in osmotic stress from 0 bar to 15 bar, germination traits like average germination percentage $(81.5 \%$ to $15 \%)$, average plumule length $(7.8 \mathrm{~cm}$ to $0.4 \mathrm{~cm})$, average no. of lateral roots (5 to 1.9), average fresh weight (124 mg to $77.4 \mathrm{mg}$ ) goes down. However, dry weight (24.7 $\mathrm{mg}$ to $40.5 \mathrm{mg}$ ) increased with increasing stress level. Average radicle length however increased at -3 bar (5.9 $\mathrm{cm})$ and -9 bar $(4.9 \mathrm{~cm})$ as compared to 0 bar $(4.4 \mathrm{~cm})$. Radicle/plumule length ratio was higher at increased stress levels i.e. 15.7 at -9 bar and 11.8 at -15 bar which decreased significantly to 1.1 at -3 bar and 0.6 at 0 bar.

Analysis of variance in the present study revealed that number of lateral roots does not varied with either of the seed priming. Also dry weight of the seedling and germination percentage was non-significant for interaction of treatment $\mathrm{x}$ osmotic stress and treatment $\mathrm{x}$ genotypes. Some of the results are in confirmation with previous findings. The present study has confirmed the previous findings of Gholamin and Khayatnezhad, 2011 on 2 wheat genotypes at 5 osmotic potential $(0$,$2,-4,-6,-8)$ and two osmopriming solutions $(\mathrm{NaCl}$ and PEG-6000) which revealed significant differences between genotypes, osmopriming solutions and osmotic potentials for fresh weight, dry weight and shoot length along with significant interactions. Decrease in germination percentage with increasing stress level was also reported by Kaur et al., 1998 in chickpea, Gholamin and Khayatnezhad, 2011 in wheat, and highest germination percentage taking place at 0 osmotic potential (Homayoun et al., 2011). Genotypes and osmotic stress levels have a significant effect on radicle and plumule length in the present study. Also genotype $\mathrm{x}$ stress level has significant differences for all the studied traits. However, Homayoun et al., 2011 reported significant genotype $\mathrm{x}$ stress level interaction for plumule length, radicle dry weight but not for radicle length.

Mean plumule length decreased with increasing stress level. Plumule length getting decreased with increasing stress level $(0,-3,-6,-9$ bar) were reported where plumule length decreased from $3.73 \mathrm{~cm}$ at 0 bar to $0.22 \mathrm{~cm}$ at -9 bar with $0.00001 \mathrm{mM}$ salicylic acid seed priming, and $4.53 \mathrm{~cm}$ at 0 bar to $0.16 \mathrm{~cm}$ at -9 bar with 0.1 $\mathrm{mM}$ salicylic acid seed priming (Movaghatian and Khorsandi, 2013). Mean radicle length for hydroprimed and $\mathrm{GA}_{3}$ treated seeds, however, increased at -3 and -9 bar stress level as compared to 0 bar (Table 2), suggesting roots searching for water. Similar kinds of results were reported (Movaghatian and Khorsandi, 2013) for lower concentrations of salicylic acid i.e. 0.00001 and $0.1 \mathrm{mM}$. At $0.00001 \mathrm{mM}$ concentration radicle length at -3 and -6 bar was $6.52 \mathrm{~cm}$ and $6.04 \mathrm{~cm}$ as compared to $4.91 \mathrm{~cm}$ at control condition ( 0 bar). Length of root was greater than length of shoot and this ratio showed an increasing trend with increasing percentage of PEG at 5\%, 10\% \& $15 \%$ (Kaur et al., 1998). In the present study also radicle/plumule length ratio was found increasing with increasing stress level and average radicle/plumule ratio was always higher for $\mathrm{GA}_{3}$ treated seed as compared to hydroprimed seeds at all stress levels (Fig. 1, Table 2). This clearly shows the ability of $\mathrm{GA}_{3}$ to increase root and shoot length over hydropriming (Abnavi and Ghobadi, 2012). There were genotypic differences for shoot length in response to hydropriming and $\mathrm{GA}_{3}$ 
treatment. However, radicle length was greater for $\mathrm{GA}_{3} @ 50 \mathrm{ppm}$ treated seeds as compared to hydroprimed seed (Abnavi and Ghobadi, 2012). Our results also showed average radicle length higher for $\mathrm{GA}_{3}$ treated as compared to hydropriming. However, genotypic difference exists for both radicle and plumule length in our study. Genotypic differences for shoot and root length were also reported (Datta et al., 2011).

In the present investigation number of lateral roots were highest at 0 bar (5.0) which lowered to 4.7 ( -3 bar), 3.2 ( -9 bar) and 1.9 at -15 bar. These results are getting confirmation from the work of Almansouri et al., 2001 reporting number of lateral roots decreasing with increasing concentration of PEG. The average fresh weight was always greater for $\mathrm{GA}_{3}$ treated seed as compared to hydroprimed seeds at all four stress levels. This clearly states that $\mathrm{GA}_{3}$ is good at mobilizing the reserves out of the seed and convert them into growing radicle and plumule (Kaur et al., 1998). Fresh weight showed a decreasing trend where it got decreased with increasing stress level for both hydroprimed and $\mathrm{GA}_{3}$ primed seed. Similar decrease in fresh weight with increasing stress level was reported by (Datta et al., 2011; Kaur et al., 1998). The average dry weight increased with increasing stress level for both hydroprimed and $\mathrm{GA}_{3}$ primed seed. This result however was in contrast to previous findings (Datta et al., 2011; Kaur et al., 1998) who reported decrease in dry weight with increasing stress level ( 0 to -8 bar). The possible reason for conflict is the methodology adopted where these workers have taken the dry weight of radicle and plumule only, whereas in this study whole seedling weight is taken for study purpose. Since increased stress level doesn't allowed seed to germinate and hence these semigerminated seed have much higher dry weight as compared to dry weight of germinated seeds.

Significant positive correlation between root and shoot length, root with root/shoot length ratio and shoot with root/shoot length ratio was reported by (Baloch et al. 2012) at 0 and 15\% PEG stress level. Root to shoot significant positive correlation at 0 bar fading away to nonsignificant correlation at -10 bars was also reported in wheat seedlings (Dhanda et al. 2004)

Among all the seven traits, germination percentage is the most important criteria for screening osmotic stress tolerant and susceptible genotypes. The overall average at all stress levels combined together revealed highest germination percentage for PBW 175 which doesn't varied significantly from C 306 and MACS 6272 but differed significantly from UP 2828 and $\mathrm{K}$ 1016. Hence, based on this germination percentage results PBW 175, C 306 and MACS 6272 can be categorized as osmotic stress tolerant and UP 2828 and K 1016 as osmotic stress susceptible genotypes.

Thus to increase the germination, application of GA3 at lower stress levels (0 to -3 bars) is recommended, while, at higher stress levels ( -9 to -15 bars) hydropriming. Thus to increase germination, priming with GA3 is recommended at lower stress levels (0 to -3 bars), whereas, hydropriming is suggested at higher stress level (-9 to -15 bars). Being said that the above said conclusion can't be generalized and genotypic variation exists for all studied germination traits which can be exploited for effective screening against drought.

\section{Acknowledgment}

Author NCG is grateful to Council of Scientific and Industrial Research (CSIR), Government of India for providing senior research fellowship (File No. 09/171(0124)/ 2012-EMR-I).

\section{References}

Abnavi, M. S. and Ghobadi, M. 2012. The Effects of Source of Priming and Post-priming Storage Duration on Seed Germination and Seedling Growth Characteristics in Wheat. Journal of Agricultural Science. 4(9): 256-268.

Ahmad, S., Ahmad, R., Ashraf, M. Y., Ashraf, M. And Waraich, E.A. 2009. Sunflower (Helianthus Annuus L.) response to drought stress at germination and seedling growth stages. Pak J 
Bot. 41: 647-654.

Almansouri, M., Kinet, J. M. and Lutts, S. 2001. Effect of salt and osmotic stresses on germination in durum wheat ( Triticum durum Desf.). Plant and Soil. 231: 243-254.

Anonymous. 2016. Progress report of All India Coordinated wheat and barley improvement project 2015-16, Director's report. Ed. G. P. Singh, ICAR-Indian Institute of wheat and barley research, Karnal, India., pp. 96.

Ashraf, C. M. and Abu-Shakra, S. 1978. Wheat seed germination under low temperature and moisture stress. Agronomy J.70: 135-139.

Baloch, M.J., Dunwell, J., Khakwani, A. A., Dennet, M., Jatoni, W. A. and Channa, S. A. 2012. Assessment of wheat cultivars for drought tolerance via osmotic stress imposed at early seedling growth stages. Journal of Agricultural Research. 50 (3): 299-310.

Bradford K. J. 1986. Manipulation of seed water relations via osmoticpriming to improve germination under stress conditions. Hortic Sci. 21: 1105-1112.

Caseiro, R., Bennett, M. A. and Marcos-Filho, J. 2004. Comparison of three priming techniques for onion seed lots differing in initial seed quality. Seed Sci Technol. 32: 365-375.

Datta, J. K., Mondal, T., Banerjee, A. and Mondal, N. K., 2011. Assessment of drought tolerance of selected wheat cultivars under laboratory condition. J. Agric. Technol. 7: 383-393.

Dhanda, S.S., Sethi, G. S. and Behl, R. K. 2004. Indices of Drought Tolerance in Wheat Genotypes at Early Stages of Plant Growth. Journal of Agronomy and Crop Science. 190(1): 6-12.

FAO. 2014. Food and Agriculture organization [Online]. Available at http://faostat3.fao.org/download/Q/QC/E [Last consulted on 1st October, 2016].

Gholamin, R. and Khayatnezhad, M. 2011. The Effects of water and salt stresses on germination in two bread wheat genotypes. African Journal of Biotechnology. 10 (77): 17805-17811.

Gupta, A. K., Singh, J., Kaur, N. and Singh, R. 1993. Effect of polyethylene glycol induced water stress on germination and reserve carbohydrate metabolism in chickpea cultivars differing in tolerance to water deficit. Plant Physiol Biochem. 31: 369-378.

Heikal, M. M., Shaddad, M. A. and Ahmed, A. M. 1982. The effect of osmotic stress on seed germination of flax, sesame and onion seeds. Biologia Plantarum. 24 (2): 124 - 129.

Homayoun, H., Daliri, M. S. and Mehrabi, P. 2011. Study of PEG stress effects on wheat (Triticum aestivum L.) cultivars at germination stage. Middle-East J. Sci. Res. 9 (1): 71-74.

Janmohammadi, M., Moradi, D. P. and Sharifzadeh, F. 2008. Seed invigoration techniques to improve germination and early growth of inbred line of maize under salinity and drought stress. Gen Appl Plant Physiol. 34: 215-226.

Kaur, S., Gupta, A. K. And Kaur, N. 1998. Gibberellic acid and kinetin partially reverse the effect of water stress on germination and seedling growth in chickpea. Plant Growth Regul. 25: 29-33.

Michel, B.E. and Kaufmann, M. R. 1973.The osmotic potential of polyethylene glycol - 6000. Plant Physiology. 51: 914-916.

Movaghatian, A. and Khorsandi, F. 2013. Effects of Salicylic Acid on Wheat Germination Parameters under Drought Stress. Am. J. Agric. Environ. Sci. 13: 1603-1608.

Rashid, A., Harris, D., Hollington, P. and Ali, S. 2004. On-farm seed priming reduces yield losses of mungbean (Vigna radiata) associated with mungbean yellow mosaic virus in the North West Frontier Province of Pakistan. Crop Prot. 23: 1119-1124.

Seo, M., Nambara, E., Choi, G. and Yamaguchi, S. 2009. Interaction of light and hormone signals in germinating seeds. Plant Mol. Biol. 69: 463-472.

Sheoran, O.P., Tonk, D. S.,Kaushik, L. S.,Hasija, R. C. and Pannu, R. S. 1998. Statistical Software Package for Agricultural Research Workers. Recent Advances in information theory, Statistics \& Computer Applications by D.S. Hooda \& R.C. Hasija, Department of Mathematics Statistics, CCS HAU, Hisar. 139-143.

\section{How to cite this article:}

Navin Chander Gahtyari, J. P. Jaiswal, Mohammed Talha, Rakesh Choudhary, Meenakshi Uniyal and Naveen Kumar. 2017. Effect of osmotic stress and seed priming on wheat seed germination traits. Int.J.Curr.Microbiol.App.Sci. 6(4): 2799-2809.

doi: https://doi.org/10.20546/ijcmas.2017.604.323 